\title{
Intensive care resources required to care for critically ill children with focal intracranial infections
}

\author{
${ }^{*}$ Marlina E. Lovett, MD,,2 Zubin S. Shah, MD, ${ }^{2}$ Melissa Moore-Clingenpeel, MA, MAS, ${ }^{3}$ \\ Eric Sribnick, MD, ${ }^{4}$ Adam Ostendorf, MD, ${ }^{2,5}$ Melissa G. Chung, MD, ${ }^{1,2,5}$ Jeffrey Leonard, MD, ${ }^{4}$ and \\ Nicole F. O'Brien, MD',2 \\ 'Division of Critical Care Medicine, Nationwide Children's Hospital; '2Department of Pediatrics, Nationwide Children's Hospital, \\ The Ohio State University College of Medicine; 'Biostatistics Core, The Research Institute, Nationwide Children's Hospital; \\ 4Division of Neurosurgery, Nationwide Children's Hospital; and 'Division of Neurology, Nationwide Children's Hospital, Columbus, \\ Ohio
}

OBJECTIVE Focal intracranial infections such as intraparenchymal abscesses or localized infections in the epidural or subdural spaces are relatively rare infections associated with both morbidity and mortality in children. Although children with these infections frequently require surgical intervention, there is a paucity of literature describing the critical care resources required to manage these cases. This retrospective chart review was performed to evaluate the resources necessary to care for critically ill children with focal intracranial infections at the authors' institution.

METHODS The authors performed a retrospective chart review of cases at their institution by using ICD-9/10 codes to identify children admitted to the pediatric intensive care unit (PICU) for either intracranial abscess or extradural and subdural abscess between 2006 and 2016. All notes, medication administration records, laboratory/imaging results, vital signs, microbiological data, and electroencephalogram results were reviewed. Data were extracted to determine the utilization of the following resources: mechanical ventilation, vasoactive medications, and intracranial pressure (ICP) monitoring. The presence of intracranial hypertension, cerebral edema, seizure, and cerebral venous sinus thrombosis were noted. Pediatric cerebral performance category (PCPC) score was determined based upon the neurological exam at discharge.

RESULTS A total of 45 children met the inclusion criteria. Their median age was 9 years (IQR 3-14 years). The incidence of focal intracranial infections in children admitted to the PICU was $0.68 / 1000$ PICU admissions in 2006 and $2.81 / 1000$ admissions in 2016. Thirty-nine children (86.7\%) underwent neurosurgical intervention. Twenty patients (44\%) required invasive mechanical ventilation, 7 (15.6\%) received vasoactive medications, and $11(24 \%)$ had an ICP monitor. Clinical seizures were detected in 12 children, including 2 who had both clinical and subclinical seizures; 1 child had subclinical seizures only. Eight children (17.8\%) developed cerebral venous sinus thrombosis. The median PCPC score was 1 (interquartile range [IQR] 1-3). The median PICU length of stay was 6.4 days (IQR 2.2-10.2 days). Children with seizures had a significantly longer hospital LOS than children without seizure.

CONCLUSIONS In this single-center, retrospective study, critical care needs for children with focal intracranial infections varied. Most frequently, patients required close neurological monitoring, and almost half required invasive mechanical ventilation. Future studies should focus on further elucidating the resources required to care for these children as well as detecting factors to identify those children most at risk for complications.

https://thejns.org/doi/abs/10.3171/2018.4.PEDS17715

KEYWORDS pediatric; intracranial abscess; subdural empyema; epidural abscess; critical care; infection

ABBREVIATIONS CI = complicated infection; CSF = cerebrospinal fluid; CVST = cerebral venous sinus thrombosis; EA = epidural abscess; EEG = electroencephalogram; HTS = hypertonic saline; IA = intraparenchymal abscess; ICD = International Classification of Disease; ICH = intracranial hypertension; ICP = intracranial pressure; IQR = interquartile range; $\mathrm{LOS}=$ length of stay; $\mathrm{PCPC}=$ pediatric cerebral performance category; $\mathrm{PICU}=$ pediatric intensive care unit; $\mathrm{SA}=$ subdural abscess; $\mathrm{SE}=$ subdural empyema.

SUBMITTED December 30, 2017. ACCEPTED April 18, 2018.

INCLUDE WHEN CITING Published online July 13, 2018; DOI: 10.3171/2018.4.PEDS17715.

* M.E.L. and Z.S.S. contributed equally to this work and share first authorship. 
$\mathrm{F}$ OCAL intracranial infections are localized collections of suppurative fluid involving the brain parenchyma, subdural space, or epidural space. ${ }^{20,21}$ These infections often have an insidious onset, originating in contiguous sources in the head or neck, and progress by direct extension. ${ }^{4,13}$ They may also arise following central nervous system seeding with bloodstream infections..$^{10,17}$ Organisms implicated in focal intracranial infections are often varied, with Streptococcus and Staphylococcus species dominating. ${ }^{2,4,5,7,19}$ Though such infections are rare, they can be life-threatening and may leave survivors with prolonged neurological deficits. ${ }^{4,10,14,19}$

There is a paucity of literature describing optimal management of children with focal intracranial infections. . $4,5,14,19$ Following medical and surgical control of the primary source, identification and management of potential complications is paramount. Septic shock, respiratory failure, seizures, cerebral edema, and cerebral venous thromboses are known complications of intracranial infections. ${ }^{1,2,4,8,16}$ Given the complexities in the management of these patients, many children will require critical care services. However, their critical care needs have not been fully investigated. ${ }^{2,14,16}$ This retrospective chart review was performed to evaluate the resources necessary to care for critically ill children with focal intracranial infections at our institution.

\section{Methods}

This study was reviewed by the Nationwide Children's Hospital institutional review board. Given the retrospective nature of the investigation, informed consent was not required. Medical records of patients treated between January 1, 2006, and December 31, 2016, were searched using International Classification of Disease (ICD) codes. Records of children ( $<18$ years of age) with either intracranial abscess (ICD-9 code 324.0, ICD-10 code G06.0) or extradural and subdural abscess unspecified (ICD-9 324.9, ICD-10 code G06.2) were included. Patients who did not require admission to the pediatric intensive care unit (PICU) were excluded. Patients were cared for in a large quaternary-level PICU.

Demographic data, medical history, and surgical history were collected. Vaccination status was classified based upon healthcare provider documentation as "up to date," "incomplete," "unvaccinated," or "unknown." Patients were classified as having a preexisting neurological history if any of the following criteria were met: seizure disorder, stroke, intracranial bleed, traumatic brain injury, neurosurgical hardware, moderate to severe developmental delay, and/or prior central nervous system infection. Notes were reviewed to determine PICU admission indication. Hospital and PICU times of admission and discharge were collected to determine length of stay (LOS). The annual average PICU LOS for all patients admitted from 2006 through 2016 was obtained from the Virtual PICU database (a quality improvement and benchmarking registry). ${ }^{3}$ Medical records were reviewed to determine the use and duration of invasive mechanical ventilation. The neurological examination performed at discharge was used to calculate the pediatric cerebral performance category (PCPC) score, a validated measure of short-term neurological outcome in children. ${ }^{9}$ Cultures of blood, urine, cerebrospinal fluid (CSF), and respiratory and intraoperative specimens were reviewed to determine causative organism(s). Neuroimaging studies within our institution are read by a pediatric radiologist. Imaging reports were reviewed, and presence of cerebral edema, cerebral herniation, or cerebral venous sinus thrombosis (CVST) was documented.

The medication administration record was used to review antibiotic regimens and the use of osmolar agents (hypertonic saline [HTS] or mannitol), corticosteroids, vasoactive medications, neuromuscular blockading agents, sedatives, antiseizure medications, and anticoagulants. Some medications have multiple clinical applications. When one of these medications was used, documentation was reviewed to determine the administration indication (e.g., benzodiazepines for sedation or for seizure control). Operative notes were reviewed to determine neurosurgical and otolaryngological management.

Comorbidities, such as elevated intracranial pressure (ICP; $\geq 20 \mathrm{~mm} \mathrm{Hg}$ ), seizure, and CVST, were noted. Peak ICP was determined from chart review. Methods to treat intracranial hypertension ( $\mathrm{ICH}$ ), including administration of osmolar agents, neuromuscular blockade, administration of pentobarbital, and hyperventilation, were noted. Seizure incidence was determined by chart review and electroencephalogram (EEG) reports. Documentation was reviewed for electrographic or clinical seizures, and clinical seizures were further described as either initially focal or generalized.

\section{Statistical Analysis}

Descriptive continuous data were reported as medians with interquartile ranges (IQRs), and categorical data were reported as percentages. Based on diagnosis codes and imaging reports, patients were subcategorized as having epidural abscess (EA), subdural empyema or subdural abscess (SE/SA), intraparenchymal abscess (IA), or a complicated infection (CI), defined as an infection involving more than 1 of the previously listed subtypes. For a comparison of categorical variables, chi-square or Fisher's exact tests were used. Mann-Whitney and Kruskal-Wallis tests were performed on continuous variables, with Dunn corrections for multiple comparisons. Linear regression was used to determine whether infection incidence changed over time. Analysis was performed based on seizure detection. Statistical significance was assumed with 2 -sided $\mathrm{p}$ values $\leq 0.05$. Statistical analysis was performed using GraphPad, Prism 7, or Microsoft Excel.

\section{Results}

\section{Demographics}

Forty-six children were included. One child treated in 2006 had incomplete documentation and was excluded from analysis, leaving a cohort of 45 children (demographics in Table 1). Based on diagnosis and imaging findings, patients were divided into those with subdural empyema/abscess (SE/SA; $n=15)$, epidural abscess (EA; $n=6$ ), intraparenchymal abscess (IA; $n=10$ ), or a complicated infection $(\mathrm{CI} ; \mathrm{n}=14)$. Median age was 9 years 
TABLE 1. Demographic information for all critically ill children with focal intracranial infections admitted to the PICU during the study period

\begin{tabular}{|c|c|c|c|c|c|}
\hline Variable & All Pts $(n=45)$ & SE/SA $(n=15)$ & $E A(n=6)$ & $\mathrm{IA}(\mathrm{n}=10)$ & $\mathrm{Cl}(\mathrm{n}=14)$ \\
\hline Median age in yrs (IQR) & $9(3-14)$ & $9(0.2-14)$ & $10.5(4.6-15)$ & $9(3.5-12.5)$ & $6(1.8-14.3)$ \\
\hline \multicolumn{6}{|l|}{ Sex } \\
\hline Male & $30(67 \%)$ & $8(53 \%)$ & $5(83 \%)$ & $6(60 \%)$ & $11(79 \%)$ \\
\hline Female & $15(33 \%)$ & $7(47 \%)$ & $1(17 \%)$ & $4(40 \%)$ & $3(21 \%)$ \\
\hline \multicolumn{6}{|l|}{ Race } \\
\hline Caucasian & $27(60 \%)$ & $8(53 \%)$ & $5(83 \%)$ & $4(40 \%)$ & $10(71 \%)$ \\
\hline African American & $11(24 \%)$ & $4(27 \%)$ & 0 & $4(40 \%)$ & $3(21 \%)$ \\
\hline Hispanic & $1(2 \%)$ & $1(7 \%)$ & 0 & 0 & 0 \\
\hline Asian & $1(2 \%)$ & $1(7 \%)$ & 0 & 0 & 0 \\
\hline Other & $5(11 \%)$ & $1(7 \%)$ & $1(17 \%)$ & $2(20 \%)$ & $1(7 \%)$ \\
\hline \multicolumn{6}{|l|}{ Vaccination status } \\
\hline Up to date & $21(47 \%)$ & $9(60 \%)$ & $3(50 \%)$ & $3(30 \%)$ & $6(43 \%)$ \\
\hline Incomplete & $6(13 \%)$ & $1(7 \%)$ & $1(17 \%)$ & $2(20 \%)$ & $2(14 \%)$ \\
\hline Not vaccinated & $4(9 \%)$ & $2(13 \%)$ & 0 & 0 & $2(14 \%)$ \\
\hline Unknown & $14(31 \%)$ & $3(20 \%)$ & $2(33 \%)$ & $5(50 \%)$ & $4(29 \%)$ \\
\hline \multicolumn{6}{|l|}{ Past medical history } \\
\hline Healthy & $17(38 \%)$ & $8(53 \%)$ & $5(83 \%)$ & $2(20 \%)$ & $2(14 \%)$ \\
\hline Neurologic history & $11(24 \%)$ & $1(7 \%)$ & $1(17 \%)$ & $3(30 \%)$ & $6(43 \%)$ \\
\hline Other conditions & $17(38 \%)$ & $6(40 \%)$ & 0 & $5(50 \%)$ & $6(43 \%)$ \\
\hline \multicolumn{6}{|l|}{ Reason for PICU admission* } \\
\hline Periop monitoring & 31 & 10 & 3 & 7 & 11 \\
\hline Seizures & 4 & 3 & 0 & 1 & 0 \\
\hline Respiratory distress & 6 & 3 & 0 & 0 & 3 \\
\hline Shock & 2 & 1 & 1 & 0 & 0 \\
\hline Anticoag & 3 & 0 & 2 & 1 & 0 \\
\hline Other & 1 & 0 & 0 & 1 & 0 \\
\hline Median PICU LOS in days (IQR) & $6.4(2.2-10.2)$ & $8.9(6.1-13.8)$ & $1.6(0.9-4)$ & $4(2.1-6.8)$ & $6(2.7-9.6)$ \\
\hline Median hospital LOS in days (IQR) & $20(12.2-30.9)$ & $29(21.2-37.5)$ & $6.8(5.1-20.6)$ & $19.9(12.8-62.7)$ & $19(8.7-22.7)$ \\
\hline Median PCPC score (IQR) & $1(1-3)$ & $2(1-3)$ & $1(1-1.8)$ & $2(1-3)$ & $2(1-4)$ \\
\hline Mortality & $1(2.2 \%)$ & 0 & 0 & 0 & $1(7 \%)$ \\
\hline
\end{tabular}

Anticoag $=$ initiation of anticoagulation; LOS $=$ length of stay; PCPC = pediatric cerebral performance category; pts = patients.

Data are number of patients (\%) unless otherwise indicated.

* Individual children may have had more than 1 reason for PICU admission.

(IQR 3-14 years). Vaccination status was up to date in $47 \%$ of children, incomplete in $13 \%$, unvaccinated in $9 \%$, and unknown in $31 \%$. The incidence of focal intracranial infections requiring intensive care was 0.68/1000 PICU admissions in 2006 and 2.81/1000 PICU admissions in 2016 (Fig. 1).

\section{Microbiological Data}

The median peak white blood cell count was $21.5 \times 10^{3} /$ $\mu \mathrm{l}\left(\mathrm{IQR} 15.6-26 \times 10^{3} / \mu \mathrm{l}\right)$. The median peak erythrocyte sedimentation rate was $56 \mathrm{~mm} / \mathrm{hr}$ (IQR 44.3-58.8 mm/ $\mathrm{hr}$ ), and the median peak C-reactive protein concentration was $7.8 \mathrm{mg} / \mathrm{dl}$ (IQR 3.5-18.8 mg/dl). Thirty-three percent of patients were bacteremic, and $22 \%$ had CSF cultures in which a pathogen was identified. In $98 \%$ of cases, a causative organism was identified on either intraoperative, blood, or CSF culture. The bacterial etiology of the fo- cal intracranial infection varied, but gram-positive bacteria were the most common. Streptococcus was the most common species, as $40 \%$ of children had culture-positive Streptococcus anginosus. Causative organisms identified in each case are available in Table 2.

\section{Imaging}

All patients underwent a head CT scan. All but 1 patient underwent MRI. Cerebral edema was present in 16 (36\%) patients (5 with SE/SA, 1 with EA, 6 with IA, and 4 with CI). Cerebral herniation was present in $3(6.7 \%)$ patients (1 patient with SE/SA, 1 with IA, and 1 with $\mathrm{CI}$ ). CVST was present in 8 patients $(17.8 \%)$ (3 with SE/SA, 2 with EA, 1 with IA, and 2 with CI).

\section{Treatment}

Broad-spectrum antibiotic therapy was initiated in all 


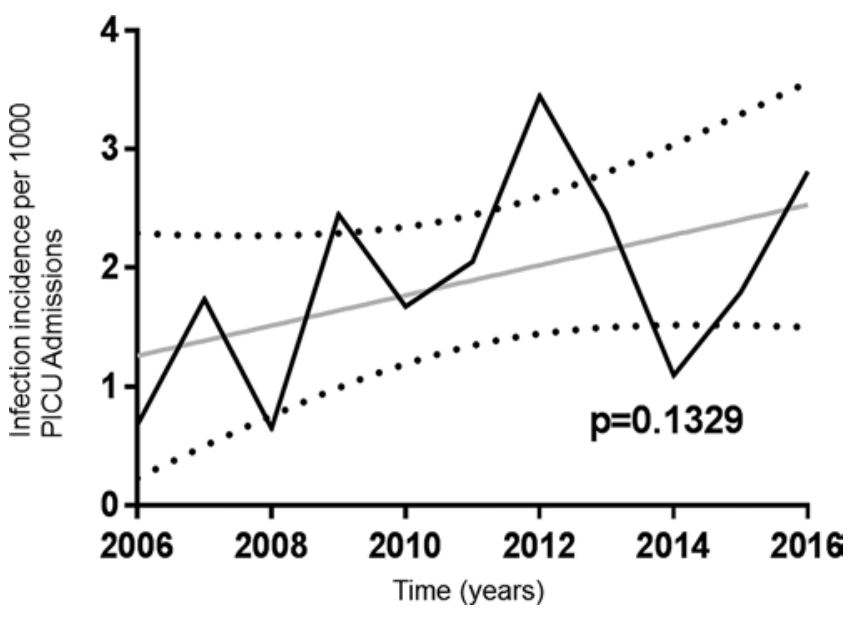

FIG. 1. Graphical representation of the incidence of focal intracranial infections requiring PICU-level care from 2006 to 2016. The gray line represents the average change over time, the dotted lines indicate the $95 \%$ confidence interval over time, and the solid black line is the actual incidence per year.

patients at the time of hospital admission or when there was clinical suspicion of infection. The initial antibiotic regimen (most commonly vancomycin, a cephalosporin, and metronidazole) covered gram-positive, gram-negative, and anaerobic organisms. In addition to broad-spectrum antibiotics, $86.7 \%$ of patients underwent neurosurgical intervention $(93 \%$ of patients with SE/SA, $50 \%$ with EA, $100 \%$ with IA, and $86 \%$ with CI) and $46.7 \%$ underwent surgical intervention by the otolaryngology team $(47 \%$ of patients with SE/SA, $83 \%$ with EA, $20 \%$ with IA, and $50 \%$ with $\mathrm{CI}$ ). The most common neurosurgical procedure was a craniotomy (29 patients; 12 with SE/SA, 3 with EA, 4 with IA, and 10 with CI); placement of burr holes was performed less frequently (6 patients; 2 with SE/SA, 2 with IA, and 2 with CI).

\section{Critical Care}

The most common reason for PICU admission in the cohort was for close perioperative neurological monitoring (Table 1). During the patients' PICU stay, resources required for their management varied. Forty-four percent of children required invasive mechanical ventilation (Table 3 ), with a median duration of 3 days (IQR 1-5.25 days). The frequency of invasive mechanical ventilation varied, yet was highest in the SE/SA group (11 children [73\%]). Noninvasive ventilation was used in $8.9 \%$ of children, with a median duration of 1 day (IQR 1-1.5 days). Vasoactive medications were used in $7(15.6 \%)$ children. Vasoactive medication use was most frequent in the SE/SA group (33\%) (Table 3). Epinephrine and norepinephrine were each used in 4 patients; 3 patients required more than 1 vasoactive medication. The most frequent indication for a vasoactive medication was hypotension, which was present in 6 of the 7 patients receiving vasoactive medications. One patient was started on a vasoactive medication specifically to maintain cerebral perfusion pressure.

Twenty-four percent of patients received an ICP monitor, most commonly an extraventricular drain (91\% of ICP monitors placed). The use of an ICP monitor was most common in the IA group (50\%), but there was no statistically significant difference with respect to location of intracranial infection (Table 3). Intracranial pressure monitors were used in $20 \%$ of children with SE/SA, $21 \%$ with $\mathrm{CI}$, and no child with EA. The median peak ICP was 25 $\mathrm{mm} \mathrm{Hg}$ (IQR 15.5-30 mm Hg). Mannitol was used for known or suspected intracranial hypertension in $3(6.7 \%)$ patients (1 patient with SE/SA, 1 with IA, and 1 with $\mathrm{CI}$ ). Hypertonic saline was used in 14 patients (31.1\%) and more HTS was used in children with SE/SA compared to all other categories $(\mathrm{p}=0.02)$. The median peak serum sodium level in children receiving hypertonic saline was $146.5 \mathrm{mmol} / \mathrm{L}$ (IQR 143.3-154.5 mmol/L). Hypertonic saline was used for the following indications: known or suspected cerebral edema (7 patients), documented intracranial hypertension (2 patients), and hyponatremia (5 patients). Additional interventions to manage intracranial hypertension included hyperventilation (4 children) and neuromuscular blockade ( 2 children). Dexamethasone was used in 14 children (31\%), and its use was more frequent in children with documented cerebral edema, but this difference was not statistically significant (OR 3.83, 95\% confidence interval 1.003-13.04, $\mathrm{p}=0.053$ ). No child with EA received dexamethasone.

The median PICU LOS for children with focal intracranial infections was 6.4 (IQR 2.2-10.2) days with a median hospital LOS of 20 days (IQR 12.2-30.9 days). When analyzed by infection subcategory, children with SE/SA had a longer PICU and hospital LOS than children with EA ( $p=0.012$ and $p=0.019$, respectively). Data obtained from the Virtual PICU database from 2006 to 2016 for all PICU admissions within our institution had a mean PICU LOS ranging from 3.04 to 3.99 days on an annual basis. The median PCPC score for children with focal intracranial infections was 1 (IQR 1-3) at the time of hospital discharge.

\section{Complications}

Seizures were detected in 13 children (28.9\%). Three presented with seizures and 10 had seizures after the initial presentation. There were no significant differences in age or the incidence of prior neurological history between children with seizures detected and those without seizures detected. Ten children with seizures had EEG monitoring ( 8 continuous, 2 routine). Five of 32 children without seizure had EEG monitoring ( 4 continuous, 1 routine). Electroencephalography was performed in children with $\mathrm{SE}$ / SA or CI; no child with EA or IA had an EEG performed. Within this context, seizures were identified in 13 (28.9\%) patients (Table 4). Detected seizures were predominantly clinical (12 children) and focal in nature (10 children). Three children (6\%) developed subclinical seizures (2 children had both clinical and subclinical seizure activity). Of the children who did not present with seizures, $52 \%$ (22/42) received seizure prophylaxis, which was evenly distributed among infection categories. Four (18\%) of the children who received seizure prophylaxis had a seizure (Table 4). Of the 18 children who received seizure prophylaxis and did not have seizures detected during hospital admission, 1 developed seizures later in life. The median 
TABLE 2. Microbiology data by year

\begin{tabular}{|c|c|c|c|c|c|c|}
\hline Year & No. of Children Cared for in PICU & Bacterial Pathogens Identified (no. of pts affected) & SA/SE $(n=15)$ & $E A(n=6)$ & $\mathrm{I}(\mathrm{n}=10)$ & $\mathrm{Cl}(\mathrm{n}=14)$ \\
\hline 2006 & 1 & Group A beta-hemolytic Streptococcus (1) & & & & $\mathrm{X}$ \\
\hline \multirow[t]{4}{*}{2007} & \multirow[t]{4}{*}{3} & Streptococcus MG intermedius (1) & & & $X$ & \\
\hline & & MRSA (1) & $X$ & & & \\
\hline & & Coagulase-negative Staphylococcus (1) & $\mathrm{X}$ & & & \\
\hline & & Group B Streptococcus (1) & $X$ & & & \\
\hline 2008 & 1 & MSSA (1) & & & $x$ & \\
\hline \multirow[t]{6}{*}{2009} & \multirow[t]{6}{*}{4} & S. anginosus (2) & & & $X$ & $X$ \\
\hline & & Treponema pallidum (1) & & & & $x$ \\
\hline & & Bacteroides fragilis (1) & & & $X$ & \\
\hline & & E. coli (1) & & & $x$ & \\
\hline & & Pseudomonas aeruginosa (1) & & & $X$ & \\
\hline & & Proprionibacterium granulosum (1) & & & $x$ & \\
\hline \multirow[t]{3}{*}{2010} & \multirow[t]{3}{*}{3} & E. coli (1) & $X$ & & & \\
\hline & & Group B Streptococcus (1) & $X$ & & & \\
\hline & & MRSA (1) & & $X$ & & \\
\hline \multirow[t]{5}{*}{2011} & \multirow[t]{5}{*}{4} & Peptostreptococcus (1) & & $x$ & & \\
\hline & & Haemophilus influenzae (1) & & $X$ & & \\
\hline & & Fusobacterium nucleatum (1) & & $X$ & & \\
\hline & & Group A beta-hemolytic Streptococcus (1) & & & $X$ & \\
\hline & & S. anginosus (1) & & & & $X$ \\
\hline \multirow[t]{10}{*}{2012} & \multirow[t]{10}{*}{7} & $P$. aeruginosa (1) & $X$ & & & \\
\hline & & S. anginosus (1) & $X$ & & & \\
\hline & & MSSA (2) & $X X$ & & & \\
\hline & & Coagulase-negative Staphylococcus (2) & $X X$ & & & \\
\hline & & MRSA (1) & & & & $X$ \\
\hline & & Peptostreptococcus (1) & & & & $X$ \\
\hline & & Enterobacter cloacae complex (1) & & & & $X$ \\
\hline & & Streptococcus viridans (1) & $X$ & & & \\
\hline & & Streptococcus pneumoniae (1) & $x$ & & & \\
\hline & & Streptococcus intermedius (1) & & & $X$ & \\
\hline \multirow[t]{4}{*}{2013} & \multirow[t]{4}{*}{6} & S. anginosus (5) & $X X X$ & $X$ & $X$ & \\
\hline & & S. pneumoniae (1) & & $X$ & & \\
\hline & & Peptostreptococcus spp. (1) & $X$ & & & \\
\hline & & Frevotella (1) & $X$ & & & \\
\hline \multirow[t]{4}{*}{2014} & \multirow[t]{4}{*}{3} & Fusobacterium (1) & $x$ & & & \\
\hline & & S. anginosus (3) & $X$ & $x$ & & $X$ \\
\hline & & Propionibacterium acnes (1) & $X$ & & & \\
\hline & & Prevotella melaninogenica (1) & & $X$ & & \\
\hline \multirow[t]{6}{*}{2015} & \multirow[t]{6}{*}{5} & MRSA (1) & $X$ & & & \\
\hline & & S. anginosus (1) & & & & $X$ \\
\hline & & $\operatorname{MSSA}(2)$ & & & & $X X$ \\
\hline & & P. acnes (1) & & & & $\mathrm{X}$ \\
\hline & & Coagulase-negative Staphylococcus (1) & $X$ & & & \\
\hline & & Group A beta-hemolytic Streptococcus (1) & $X$ & & & \\
\hline
\end{tabular}

CONTINUED ON PAGE 458 "

duration of seizure prophylaxis for the 17 children without seizure was 49 days (IQR 10-68 days). Six children $(30 \%)$ without prophylaxis $(n=20)$ had a seizure (Fig. 2). Children with identified seizures had a higher rate of endotracheal intubation $(69 \%)$ than those without seizure $(34 \%, \mathrm{p}=0.049)$. Children with identified seizures had a nonsignificant increase in PICU LOS compared to those without seizure (8.7 vs 4.7 days, $\mathrm{p}=0.18$ ). Hospital LOS 
Lovett et al.

» CONTINUED FROM PAGE 457

TABLE 2. Microbiology data by year

\begin{tabular}{|c|c|c|c|c|c|c|}
\hline Year & No. of Children Cared for in PICU & Bacterial Pathogens Identified (no. of pts affected) & SA/SE (n = 15) & $E A(n=6)$ & $I A(n=10)$ & $\mathrm{Cl}(\mathrm{n}=14)$ \\
\hline \multirow[t]{8}{*}{2016} & \multirow[t]{8}{*}{8} & P. melaninogenica (1) & $\mathrm{X}$ & & & \\
\hline & & Eikenella corrodens (1) & $\mathrm{X}$ & & & \\
\hline & & S. anginosus (5) & $\mathrm{X}$ & $\mathrm{X}$ & $X X X$ & \\
\hline & & P. acnes (2) & $X X$ & & & \\
\hline & & Mycoplasma hominis (1) & $\mathrm{X}$ & & & \\
\hline & & Toxoplasma gondii (1) & & & & $\mathrm{X}$ \\
\hline & & H. influenzae (1) & & & & $\mathrm{X}$ \\
\hline & & Group A beta-hemolytic Streptococcus (1) & & & & $X$ \\
\hline
\end{tabular}

MRSA = methicillin-resistant $S$. aureus; $M S S A=$ methicillin-sensitive $S$. aureus.

Each $X$ indicates a single case of infection with the specified organism. Some children were infected with more than 1 bacterial pathogen.

was significantly longer in children with seizures than in children without seizures ( 28 vs 19 days, $p=0.02$ ). There was no significant difference in PCPC scores based on seizure detection.

Eight children (17.8\%) with focal intracranial infections developed CVST. The incidence of thrombosis was similar among infection subcategories (Table 3). Four children had isolated superior sagittal sinus thromboses, 1 child had thrombosis in the superior ophthalmic vein and cavernous sinus, 1 child had thrombosis in the transverse and sigmoid sinuses, 1 child had thrombosis in the superior sagittal si- nus and the cavernous sinus, and 1 child had diffuse thrombosis throughout the superior sagittal sinus, transverse and sigmoid sinuses, straight sinus, and vein of Galen. Of children with documented thromboses on imaging, 3 (37.5\%; 2 children with EA and 1 child with IA) received anticoagulation therapy, initially with a heparin infusion. The remainder of the children with documented thromboses did not receive anticoagulation therapy or a surgical thrombectomy, nor was any child found to have clot progression on repeat imaging. All children with CVST received fluid balance monitoring and maintained euvolemia.

TABLE 3. Complications and critical care resources utilized based upon infection subcategory

\begin{tabular}{|c|c|c|c|c|c|}
\hline Parameter & SE/SA $(n=15)$ & $E A(n=6)$ & $\mathrm{I}(\mathrm{n}=10)$ & $\mathrm{Cl}(\mathrm{n}=14)$ & p Value* \\
\hline Endotracheal intubation & $11(73 \%)$ & $2(33 \%)$ & $3(30 \%)$ & $4(29 \%)$ & $0.054 \dagger$ \\
\hline Duration of invasive mechanical ventilation in days, median (IQR) & $4(1-7)$ & $1(1-1)$ & $1(1-2)$ & $5(3.5-11.75)$ & $0.045 \ddagger$ \\
\hline Noninvasive ventilation & $3(20 \%)$ & 0 & 0 & $1(7 \%)$ & $0.27 \dagger$ \\
\hline Vasoactive medication used & $5(33 \%)$ & $1(17 \%)$ & 0 & $1(7 \%)$ & $0.10 \dagger$ \\
\hline ICP monitor & $3(20 \%)$ & 0 & $5(50 \%)$ & $3(21 \%)$ & $0.13 \dagger$ \\
\hline Peak ICP & $30,30(n=2) \S$ & NA & $14,20(n=2) \S$ & $12,31(n=2) \S$ & NA \\
\hline Mannitol use & $1(7 \%)$ & 0 & $1(10 \%)$ & $1(7 \%)$ & $0.89 \dagger$ \\
\hline HTS use & $9(60 \%)$ & 0 & $2(20 \%)$ & $3(21 \%)$ & $0.02 \dagger$ \\
\hline Dexamethasone use & $4(26.7 \%)$ & 0 & $5(50 \%)$ & $5(35.7 \%)$ & $0.20 \dagger$ \\
\hline Hyperventilation & $2(13 \%)$ & 0 & $1(10 \%)$ & $1(7 \%)$ & $0.80 \dagger$ \\
\hline Neuromuscular blockade for elevated ICP & $2(13 \%)$ & 0 & 0 & 0 & $0.24 \dagger$ \\
\hline Pentobarbital for elevated ICP & 0 & 0 & 0 & $1(7 \%)$ & $0.52 \dagger$ \\
\hline Cerebral edema & $5(33 \%)$ & $1(17 \%)$ & $6(60 \%)$ & $4(29 \%)$ & $0.28 \dagger$ \\
\hline Cerebral herniation & $1(7 \%)$ & 0 & $1(10 \%)$ & $1(7 \%)$ & $0.89 \dagger$ \\
\hline CVST & $3(20 \%)$ & $2(33 \%)$ & $1(10 \%)$ & $2(14 \%)$ & $0.67 \dagger$ \\
\hline PICU LOS in days, median (IQR) & $8.9(6.1-13.8)$ & $1.6(0.9-4)$ & $4(2.1-6.8)$ & $6(2.7-9.6)$ & $0.011 \ddagger$ \\
\hline Hospital LOS in days, median (IQR) & $29(21.2-37.5)$ & $6.8(5.1-20.6)$ & $19.9(12.8-62.7)$ & $19(8.7-22.7)$ & $0.014 \ddagger$ \\
\hline PCPC score, median (IQR) & $2(1-3)$ & $1(1-1.8)$ & $2(1-3)$ & $2(1-4)$ & $0.41 \ddagger$ \\
\hline
\end{tabular}

Data are number of patients (\%) unless otherwise indicated. Boldface type indicates statistical significance.

* The $p$ values shown are based on subcategory comparisons. For PICU LOS, using Dunn's multiple comparisons test, when subdural was compared to epidural, $p=$

0.012. For hospital LOS, using Dunn's multiple comparisons test, when subdural was compared to epidural, $p=0.019$.

$\ddagger$ Based on Kruskal-Wallis test.

† Based on chi-square test.

$\S$ For peak ICP data, the $n$ shown is the number available with ICP data documented. 
TABLE 4. Seizure differences among the cohort

\begin{tabular}{|c|c|c|c|c|c|}
\hline Parameter & SE/SA $(n=15)$ & $E A(n=6)$ & $\mathrm{IA}(\mathrm{n}=10)$ & $\mathrm{Cl}(\mathrm{n}=14)$ & p Value \\
\hline Presented w/ Sz & 2 & 0 & 0 & 1 & $0.53^{*}$ \\
\hline EEG use & $11(73 \%)$ & 0 & 0 & $4(29 \%)$ & $0.0003 \uparrow$ \\
\hline Sz prophylaxis & $7(54 \%)$ & $3(50 \%)$ & $5(50 \%)$ & $7(54 \%)$ & $0.97 \dagger$ \\
\hline Sz after presentation & $5(38 \%)$ & 0 & $1(10 \%)$ & $4(31 \%)$ & $0.18 \dagger$ \\
\hline Received prophylaxis \& had Sz & $2 / 7(28.6 \%)$ & $0 / 3$ & $0 / 5$ & $2 / 7(28.9 \%)$ & $0.42 \dagger$ \\
\hline No prophylaxis \& had Sz & $3 / 6(50 \%)$ & $0 / 3$ & $1 / 5(20 \%)$ & $2 / 6(33.3 \%)$ & $0.44 \dagger$ \\
\hline
\end{tabular}

\section{Discussion}

Focal intracranial infections are relatively uncommon in children but are associated with prolonged, complicated hospital courses requiring multiple interventions. Most literature has focused on surgical management and suggests that these patients benefit from prompt initiation of antimicrobial therapy as well as surgical evacuation of focal lesions. There is a paucity of literature describing the ICU resources required to care for these patients. To our knowledge, this is the first in-depth exploration of this topic.

Our single-center, retrospective analysis suggests that the number of children with focal intracranial infections treated within our PICU is increasing. In 2006, 1 child with a focal intracranial infection required PICU admission compared to 8 children in 2016. Given the retrospective nature of this study, it is difficult to determine whether the rising incidence of PICU admission reflects a higher acuity or a higher incidence of focal intracranial infection. A study of children with brain abscesses noted a slightly higher incidence over time (an increase from 1.03 to 1.09 cases/5000 admissions in 1989-1999 compared to 20002009). ${ }^{19}$ Similarly, an increase in the annual admission rate for children with sinogenic intracranial abscesses has been described from 1997 to 2006 (3 vs 4.04 admissions/106 children per year). ${ }^{18}$ This could be further clarified in a prospective study evaluating the incidence of all focal intracranial infections and the incidence of those requiring PICU admission. Our PICU LOS is prolonged, with a median duration of 6.4 days, compared to our institutional average of 3 days for all PICU patients. Despite the prolonged LOS, these children did well (median PCPC score of 1). The most frequent bacterial etiology for our cohort was Streptococcus species, consistent with prior studies. ${ }^{5,14}$

Interestingly, $67 \%$ of children in our study were male. This is in agreement with a prior systematic review of the literature which found that $70 \%$ of subjects with intracranial complications of sinusitis were male. ${ }^{17}$ The reason for the varying incidence between the sexes is unknown but has been noted in both developed and developing countries. ${ }^{13,14,16,17}$ We hypothesize that this could be due to hormonal differences.

Perioperative monitoring was the predominant indication for PICU admission, which is not surprising given that surgical intervention is a mainstay of treatment. Within our cohort, the patients in the EA group had the lowest rate of neurosurgical intervention at 50\% compared to $93 \%$ in the SE/SA group and $100 \%$ in the IA group. Overall, $86.7 \%$ of our cohort underwent neurosurgical intervention and $46.7 \%$ underwent otolaryngological intervention, consistent with the report by Brouwer et al. of neurosurgical intervention in $87 \%$ of patients with brain abscesses. ${ }^{5}$ Therefore, it is reasonable to assume that in these children, following invasive surgical procedures, close neurological monitoring is required in the critical care setting as these children are at risk for rapid neurological deterioration.

When all categories of our study are evaluated, the patients in the SE/SA group appear to have had the highest illness severity. This cohort had the highest incidence of intubation and use of vasoactive medications. Although ICP monitors were used in only $20 \%$ of the SE/SA group, the SE/SA cohort had the highest rate of HTS administration. In addition, seizures were detected more frequently in these patients than in those in the EA, IA, or CI groups. Finally, patients in the SE/SA group had the most prolonged PICU and hospital LOS.

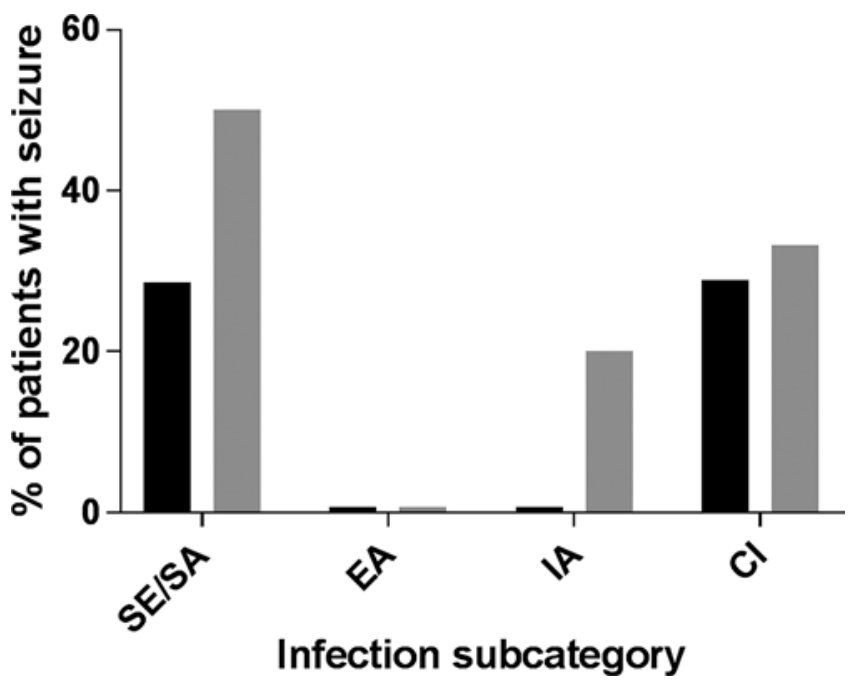

FIG. 2. Administration of seizure prophylaxis and seizure incidence. Black bars represent patients who received prophylaxis and gray bars represent patients who did not. 
Forty-four percent of the cohort required endotracheal intubation, with a 3-day median duration of mechanical ventilation. The reasons for prolonged respiratory failure are unclear, though in some cases it is likely to have been secondary to shock (15\% received vasoactive medications) or intracranial hypertension $(31 \%$ had cerebral edema, $24.4 \%$ had ICP monitors). The retrospective nature of this study prevented definitive determination of the requirement for mechanical ventilation, but future prospective studies should evaluate this.

Our data suggest that intracranial hypertension is not an uncommon complication in children with focal intracranial infections. The median documented peak ICP in our cohort was $25 \mathrm{~mm} \mathrm{Hg}$. Thirty-one percent of the patients received HTS, and 6.7\% received mannitol. Hyperventilation and neuromuscular blockade were used infrequently. Dexamethasone was used in $31.1 \%$ of patients (all categories except the EA cohort), and though there was a trend toward more frequent use in those with cerebral edema, this was not statistically significant. The use of corticosteroids in our cohort was lower than the previously reported use in 55\% of patients with brain abscesses previously reported by Brouwer et al..$^{5}$ This may reflect the heterogeneity of our cohort; if dexamethasone use in only our IA group is considered, the rate is similar to the previously reported rate of 55\%. The indication for use of dexamethasone was unclear. In addition, there is a lack of highquality evidence supporting the use of corticosteroids in focal intracranial infections, particularly since corticosteroids may inhibit antimicrobial penetration..$^{11}$ The authors of one study have suggested that it may be reasonable to consider corticosteroid use perioperatively to reduce ICH and avoid herniation. ${ }^{2}$ Future studies could investigate the systematic use of dexamethasone to better characterize the patient population, including location of intracranial infection, which may benefit from corticosteroids. Furthermore, future studies should focus on most effective management strategies for children with focal intracranial infections and ICH.

Seizures were neither a common presenting symptom nor the predominant reason for PICU admission. Three children $(6.6 \%, 2$ with SE/SA and 1 with CI) presented with seizure activity, which is a lower rate than the $18 \%-$ $29 \%$ previously reported.,17 The patients with seizures in our study were all given treatment-dose antiseizure medications. Fifty-two percent of the cohort received seizure prophylaxis, distributed evenly among all categories (54\% in the SE/SA group alone), which was lower than the $69 \%$ utilization rate reported in a study of subdural empyema patients. ${ }^{10}$ EEGs were obtained in 15 children (33\% of the cohort, and solely in patients with SE/SA or CI). The American Clinical Neurophysiology Society consensus statement, released in 2015, recommends continuous EEG monitoring for individuals in the ICU with altered mental status and intracranial infection..$^{12}$ The infrequent use of EEG in our cohort may have been due to the number of patients with normal mental status admitted for postoperative monitoring. It is reasonable to have a low threshold to obtain EEG if a child develops altered mental status, particularly a child with SE/SA or CI.

Seizures were identified in 13 children and were pre- dominantly clinical and focal in nature. Seizures were detected in $47 \%$ of the SE/SA group, higher than the previously reported 20\%-40\% incidence. ${ }^{6,15}$ Interestingly, seizures were most frequent in the SE/SA and CI groups; no child in the EA group had a seizure. When all categories were combined, seizures were more common in children who did not receive prophylaxis $(30 \%)$ than in those who received prophylaxis $(18 \%, \mathrm{p}=0.44)$. This difference was more striking in the SE/SA group, as the incidence of seizure in those patients with prophylaxis was $28.6 \%$ compared to $50 \%$ in those without. Though this was not statistically significant, the sample size was small. Despite the relatively high frequency of seizures in children with focal intracranial infections, there is a lack of evidencebased guidelines regarding seizure prophylaxis. Seizure occurrence was associated with a more prolonged hospital stay, but it remains unclear whether this is a modifiable factor or seizures simply occurred more often in children with severe infections who were hospitalized longer for the management of their primary disease. In the future, we propose investigating the utility of seizure prophylaxis as well as the optimal agent and dosage to minimize seizures and the impact of this management on hospital LOS.

CVSTs have a known association with head and neck infections. Recent studies of adults with CVST estimate $6 \%-12 \%$ of patients had head and neck infections as a precipitating factor. ${ }^{22}$ In children, DeVeber et al. found that $18 \%$ of children with CVST had a head or neck infection, although infection type was not specified. ${ }^{8}$ Within our cohort, 8 patients (17.8\%) developed CVST, which was relatively evenly distributed between categories, and 3 patients received anticoagulation. In the literature, recommendations regarding the use of anticoagulation in this setting are unclear. In 57 children with head and neck infections with CVST, there was no significant difference in the incidence of poor outcome between those who received anticoagulation and those who did not. ${ }^{22}$ Within our cohort, $75 \%$ of children with CVST underwent neurosurgical intervention. All children with CVST received fluid balance monitoring and maintained euvolemia. No child within our cohort had evidence of clot propagation on repeat imaging. Therefore, the decision to initiate anticoagulation is one that requires thoughtful interdisciplinary discussion. It may be reasonable to clinically monitor these children, treat them supportively (rehydration, appropriate antibiotic therapy), pursue serial head imaging, and initiate anticoagulation if there is clot propagation. This would need to be weighed against the risk of anticoagulation in a child who may need repeated neurosurgical interventions.

Our study has several limitations. First, we are limited by the retrospective nature of this study. Although we tried to perform extraction of the medical record in a systematic manner, it is possible that some children were omitted. One child treated during the early part of our investigative period had incomplete documentation, and necessary data were missing; therefore, his case was excluded from the study. This review is limited by the quality of the data. Some data were extrapolated from physician documentation and flowsheets, which may be incomplete. In particular, granular ICP data were missing for 5 patients, and ICP data were obtained from physician or nursing notes. 
Head imaging and EEG results were dependent upon the radiologist's or neurologist's interpretation and were not reviewed by an independent observer. Our sample size was small, which limited our ability to detect a significant difference between categories. Due to the retrospective nature of this study, PCPC scores were calculated based on documentation at discharge, and long-term outcomes were not available.

\section{Conclusions}

Children with focal intracranial infections are likely to require critical care services during their hospital stay. Many receive mechanical ventilation, vasoactive medications, and intensive therapies to manage intracranial hypertension. These children are at risk to develop complications, such as seizures and CVST. Future studies should focus on prospective characterization of critical care needs and documentation of frequency, severity, and management of important secondary complications. Furthermore, seizure prophylaxis, particularly in children with SE/SA or $\mathrm{CI}$, should be investigated in a prospective manner to determine the utility as well as optimal duration and medication. These data are imperative to develop high-quality management guidelines for these children.

\section{References}

1. Abend NS, Arndt DH, Carpenter JL, Chapman KE, Cornett KM, Gallentine WB, et al: Electrographic seizures in pediatric ICU patients: cohort study of risk factors and mortality. Neurology 81:383-391, 2013

2. Alvis Miranda H, Castellar-Leones SM, Elzain MA, Moscote-Salazar LR: Brain abscess: current management. J Neurosci Rural Pract 4 (Suppl 1):S67-S81, 2013

3. Bennett TD, Spaeder MC, Matos RI, Watson RS, Typpo KV, Khemani RG, et al: Existing data analysis in pediatric critical care research. Front Pediatr 2:79, 2014

4. Bonfield CM, Sharma J, Dobson S: Pediatric intracranial abscesses. J Infect 71 (Suppl 1):S42-S46, 2015

5. Brouwer MC, Coutinho JM, van de Beek D: Clinical characteristics and outcome of brain abscess: systematic review and meta-analysis. Neurology 82:806-813, 2014

6. Bruner DI, Littlejohn L, Pritchard A: Subdural empyema presenting with seizure, confusion, and focal weakness. West J Emerg Med 13:509-511, 2012

7. Cole TS, Clark ME, Jenkins AJ, Clark JE: Pediatric focal intracranial suppuration: a UK single-center experience. Childs Nerv Syst 28:2109-2114, 2012

8. deVeber G, Andrew M, Adams C, Bjornson B, Booth F, Buckley DJ, et al: Cerebral sinovenous thrombosis in children. N Engl J Med 345:417-423, 2001

9. Fiser DH: Assessing the outcome of pediatric intensive care. J Pediatr 121:68-74, 1992

10. French H, Schaefer N, Keijzers G, Barison D, Olson S: Intracranial subdural empyema: a 10-year case series. Ochsner J 14:188-194, 2014

11. Hakan T, Ceran N, Erdem I, Berkman MZ, Göktaş P: Bacterial brain abscesses: an evaluation of 96 cases. J Infect 52:359-366, 2006

12. Herman ST, Abend NS, Bleck TP, Chapman KE, Drislane FW, Emerson RG, et al: Consensus statement on continuous EEG in critically ill adults and children, part I: indications. J Clin Neurophysiol 32:87-95, 2015
13. Isaacson B, Mirabal C, Kutz JW Jr, Lee KH, Roland PS: Pediatric otogenic intracranial abscesses. Otolaryngol Head Neck Surg 142:434-437, 2010

14. Laulajainen-Hongisto A, Lempinen L, Färkkilä E, Saat R, Markkola A, Leskinen K, et al: Intracranial abscesses over the last four decades; changes in aetiology, diagnostics, treatment and outcome. Infect Dis (Lond) 48:310-316, 2016

15. Nathoo N, Nadvi SS, van Dellen JR, Gouws E: Intracranial subdural empyemas in the era of computed tomography: a review of 699 cases. Neurosurgery 44:529-536, 1999

16. Ndubuisi CA, Ohaegbulam SC, Mezue WC, Chikani MC, Nkwerem SP, Ozor II: Management of brain abscess: changing trend and experience in Enugu, Nigeria. Niger J Surg 23:106-110, 2017

17. Patel NA, Garber D, Hu S, Kamat A: Systematic review and case report: intracranial complications of pediatric sinusitis. Int J Pediatr Otorhinolaryngol 86:200-212, 2016

18. Piatt JH Jr: Intracranial suppuration complicating sinusitis among children: an epidemiological and clinical study. $\mathbf{J}$ Neurosurg Pediatr 7:567-574, 2011

19. Shachor-Meyouhas Y, Bar-Joseph G, Guilburd JN, Lorber A, Hadash A, Kassis I: Brain abscess in children-epidemiology, predisposing factors and management in the modern medicine era. Acta Paediatr 99:1163-1167, 2010

20. Tunkel AR: Brain abscess, in Bennett J, Dolin R, Blaser M (eds): Mandell, Douglas, and Bennett's Principles and Practice of Infectious Diseases, ed 8. Philadelphia: Elsevier, 2015, pp 1164-1176

21. Tunkel AR: Subdural empyema, epidural abscess, and suppurative intracranial thrombophlebitis, in Bennett $\mathrm{J}$, Dolin R, Blaser M (eds): Mandell, Douglas, and Bennett's Principles and Practice of Infectious Diseases, ed 8. Philadelphia: Elsevier, 2015, pp 1177-1185

22. Zuurbier SM, Coutinho JM, Stam J, Canhão P, Barinagarrementeria F, Bousser MG, et al: Clinical outcome of anticoagulant treatment in head or neck infection-associated cerebral venous thrombosis. Stroke 47:1271-1277, 2016

\section{Disclosures}

The authors report no conflict of interest concerning the materials or methods used in this study or the findings specified in this paper.

\section{Author Contributions}

Conception and design: Lovett, O'Brien. Acquisition of data: Lovett, Shah. Analysis and interpretation of data: Lovett, MooreClingenpeel, Sribnick, Ostendorf, Leonard, O’Brien. Drafting the article: Lovett, Shah. Critically revising the article: Lovett, Shah, Moore-Clingenpeel, Sribnick, Ostendorf, Leonard, O'Brien. Reviewed submitted version of manuscript: all authors. Approved the final version of the manuscript on behalf of all authors: Lovett. Statistical analysis: Lovett, Moore-Clingenpeel.

\section{Supplemental Information}

\section{Previous Presentations}

A portion of this work has been previously presented in abstract and poster form at the Nationwide Children's Hospital Research Retreat.

\section{Correspondence}

Marlina E. Lovett: Nationwide Children's Hospital, Columbus, OH. marlina.lovett@nationwidechildrens.org. 